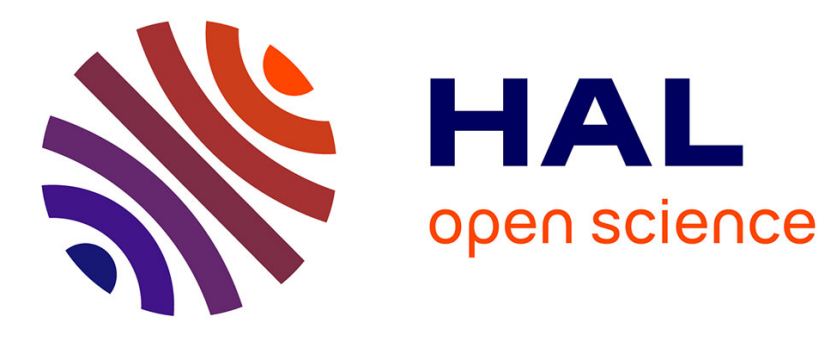

\title{
Interacting Cracks Obey a Multiscale Attractive to Repulsive Transition
}

\author{
Marie-Émeline Schwaab, Thierry Biben, Stéphane Santucci, Anthony \\ Gravouil, Loïc Vanel
}

\section{To cite this version:}

Marie-Émeline Schwaab, Thierry Biben, Stéphane Santucci, Anthony Gravouil, Loïc Vanel. Interacting Cracks Obey a Multiscale Attractive to Repulsive Transition. Physical Review Letters, 2018, 120 (25), pp.255501. 10.1103/PhysRevLett.120.255501 . hal-02289858

\section{HAL Id: hal-02289858 \\ https://univ-lyon1.hal.science/hal-02289858}

Submitted on 19 Jan 2022

HAL is a multi-disciplinary open access archive for the deposit and dissemination of scientific research documents, whether they are published or not. The documents may come from teaching and research institutions in France or abroad, or from public or private research centers.
L'archive ouverte pluridisciplinaire HAL, est destinée au dépôt et à la diffusion de documents scientifiques de niveau recherche, publiés ou non, émanant des établissements d'enseignement et de recherche français ou étrangers, des laboratoires publics ou privés. 


\title{
Interacting Cracks Obey a Multiscale Attractive to Repulsive Transition
}

\author{
Marie-Émeline Schwaab, ${ }^{1}$ Thierry Biben, ${ }^{1}$ Stéphane Santucci, ${ }^{2,3}$ Anthony Gravouil, ${ }^{4}$ and Loïc Vanel ${ }^{1, *}$ \\ ${ }^{1}$ Université de Lyon, Université Claude Bernard Lyon 1, CNRS, Institut Lumière Matière, F-69622 Villeurbanne, France \\ ${ }^{2}$ Université de Lyon, ENS de Lyon, Université Claude Bernard, CNRS, Laboratoire de Physique, F-69342 Lyon, France \\ ${ }^{3}$ Lavrentyev Institute of Hydrodynamics, Novosibirsk, Russia \\ ${ }^{4}$ Université de Lyon, LaMCoS, INSA-Lyon, CNRS UMR5259, F-69621 Lyon, France
}

(Received 13 February 2018; published 20 June 2018)

\begin{abstract}
The observed repulsive behavior of two initially collinear cracks growing towards each other and leading to a hook-shaped path questioned recently the validity of the principle of local symmetry within linear elastic fracture mechanics theory. Our theoretical and numerical work solves this dilemma, providing the precise geometric conditions for the existence of this repulsive phase. We moreover reveal a multiscale behavior of the repulsive-attractive transition, explaining its ubiquitous occurrence, but also the difficulty to predict the final cracks' paths.
\end{abstract}

DOI: 10.1103/PhysRevLett.120.255501

Because any crack tip acts as a singularity that alters nonlocally the stress field, multiple cracks affect each other, curving to form complex paths and fracture patterns [1]. The particular case of en passant crack pairs [2], two parallel and offset cracks approaching each other by propagating through their inner tips, is commonly observed and sometimes exhibits a truly counterintuitive behavior: the cracks initially curve away from each other before turning sharply inwards and attracting until coalescence, forming a hook-shaped path. This supposedly universal behavior has been observed in a wide variety of materials, both soft [3,4] and brittle [2,5,6], and a wide variety of scales, ranging from the micrometric scale in thin metallic films or biological tissues [7] to the geological scale in planetary crust or pack ice $[8,9]$.

Yet, to the best of our knowledge, the repulsive component of the paths has been seldom studied theoretically. Fender et al. [10] presented a simple geometric model fitting their experimental data, but corresponding only to an attractive trajectory. Contrary to the attractive phase, the repulsive phase is not systematically present and the precise conditions necessary for its appearance are still unclear. Melin [11] explained the repulsion of two aligned cracks as an instability due to microscopic defects in the materials, but did not make any predictions of the repulsion magnitude, or extend the study to the case of offset cracks. Linear elastic fracture mechanics is the theoretical framework of reference for the fracture of brittle materials and needs to be completed by a bifurcation criterion to predict the propagation direction and the final crack path shape. Using the uncommon strain-energy density criterion [12], Gdoutos [13] determined angles of repulsion in some examples, but a systematic analysis to determine the geometric conditions to induce repulsion remains lacking. Using the more standard principle of local symmetry [14,15], Ghelichi and Kamrin [16] presented a set of analytical tools to study en passant cracks but did not explicitly study the repulsive part of en passant cracks paths. Furthermore, a striking discrepancy between theory and experiments was pointed out by Dalbe et al. [17]: while maximum repulsion seems to happen for perfectly aligned cracks, theory using the principle of local symmetry criterion predicts no deflection in this case. A recent study even suggested that plasticity may be needed to induce repulsion [18]. Therefore, current state of the art gives conflicting results and clearly calls for a thorough re-examination of the linear elastic fracture mechanics framework when applied to the case of en passant cracks.

In this Letter, combining theoretical and numerical analysis, we show that linear elasticity associated with the standard criterion of crack propagation (the principle of local symmetry) explains the existence of crack repulsion as well as some of the discrepancies with experimental observations $[3,6,17]$. We unveil the power law dependency of the geometric conditions controlling the value of the initial bifurcation angle, providing a broad insight as to which en passant crack pairs will initially attract or repel one another. Our results emphasize the multiscale nature of the en passant problem; the length scales characterizing the transition from attraction to repulsion may be orders of magnitude smaller than the crack length. This will cause a high sensitivity of crack trajectories with respect to their precise location, making it possible for cracks or faults to produce very different and hard to predict patterns as they grow closer to each other.

In $2 \mathrm{D}$, the stress distribution around the tips is entirely defined by the stress intensity factors [1] $K_{I}$ and $K_{I I}$, two parameters quantifying the relative importance of the two cracking modes (opening and sliding respectively). In this Letter, we differentiate $K_{I}$ and $K_{I I}$, the stress intensity 
factors in the original fracture problem, from $K_{I}^{*}$ and $K_{I I}^{*}$, the stress intensity factors at the tips of an infinitesimally small crack extension. The most commonly used criteria to determine the direction of crack growth in a homogeneous and isotropic material are the maximum hoop stress [19], the maximum energy release rate [20], and the principle of local symmetry $[14,15]$. While all these criteria give similar results and can be considered equivalent, the principle of local symmetry is often considered as more physically acceptable for cracks propagating into homogeneous and isotropic materials [21]. We choose, therefore, the principle of local symmetry criterion stating that all cracks turn to propagate into pure opening mode, which translates into the equation $K_{I I}^{*}=0$.

The geometry of en passant cracks [Fig. 1(a)] is controlled by three parameters: the half crack length $L_{f}$, and the two tip to tip separation distances $2 \delta x$ and $2 \delta y$. In arbitrary units, $L_{f}$ was taken between 1 and 200, while $\delta x$ and $\delta y$ covered $\pm[0 ; 100]$ and $[0 ; 300]$, respectively, in order to chart the $(\Delta x, \Delta y)$ space with over 7000 computation points. We are always considering a far-field uniaxial stress field in the direction orthogonal to the cracks. To determine the angle $\theta$ formed between the original crack and its extension satisfying the principle of local symmetry, we determine first $K_{I I}^{*}$ using universal polynomial expressions depending only on $\theta, K_{I}$, and $K_{I I}$ [21]. $K_{I}$ and $K_{I I}$ can be easily determined in a finite element computation through the well-known interaction integral procedure [22-24]. Therefore, our scheme to determine $\theta$ amounts to two steps: a finite element analysis to compute $K_{I}$ and $K_{I I}$ and a simple minimization algorithm solving $K_{I I}^{*}(\theta)=0$. We were able to validate this computational procedure by

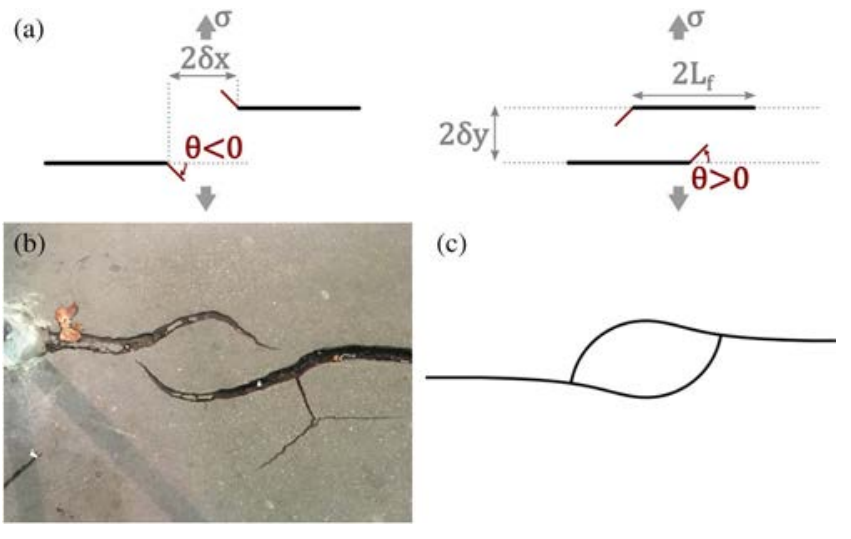

FIG. 1. (a) En passant crack configurations in the approaching and partially superimposed cases. The behavior is repulsive when $\theta<0$ and attractive when $\theta<0$. The cracks are always submitted to a far-field uniaxial tension. (b) Real life example of approaching en passant cracks in asphalt concrete. The case of superimposed en passant cracks is most often observed at the microscale; see for example in bones [26], stretchable electronics [27], or mechanical sensors [28]. (c) Simulated en passant crack paths using our iterated scheme. comparing quantitatively some of our results with the ones obtained using an analytical procedure predicting the stress intensity factors of interacting cracks [16]. The specifics concerning the boundary value problem, the computation scheme, and the validation of our procedure are available in the Supplemental Material [25].

We find that the problem reduces to a set of only two parameters: the scaled tip to tip relative separation $\Delta x=$ $\delta x / L_{f}$ and $\Delta y=\delta y / L_{f}$. The efficiency and robustness of calculation allowed us to repeat the computation for many
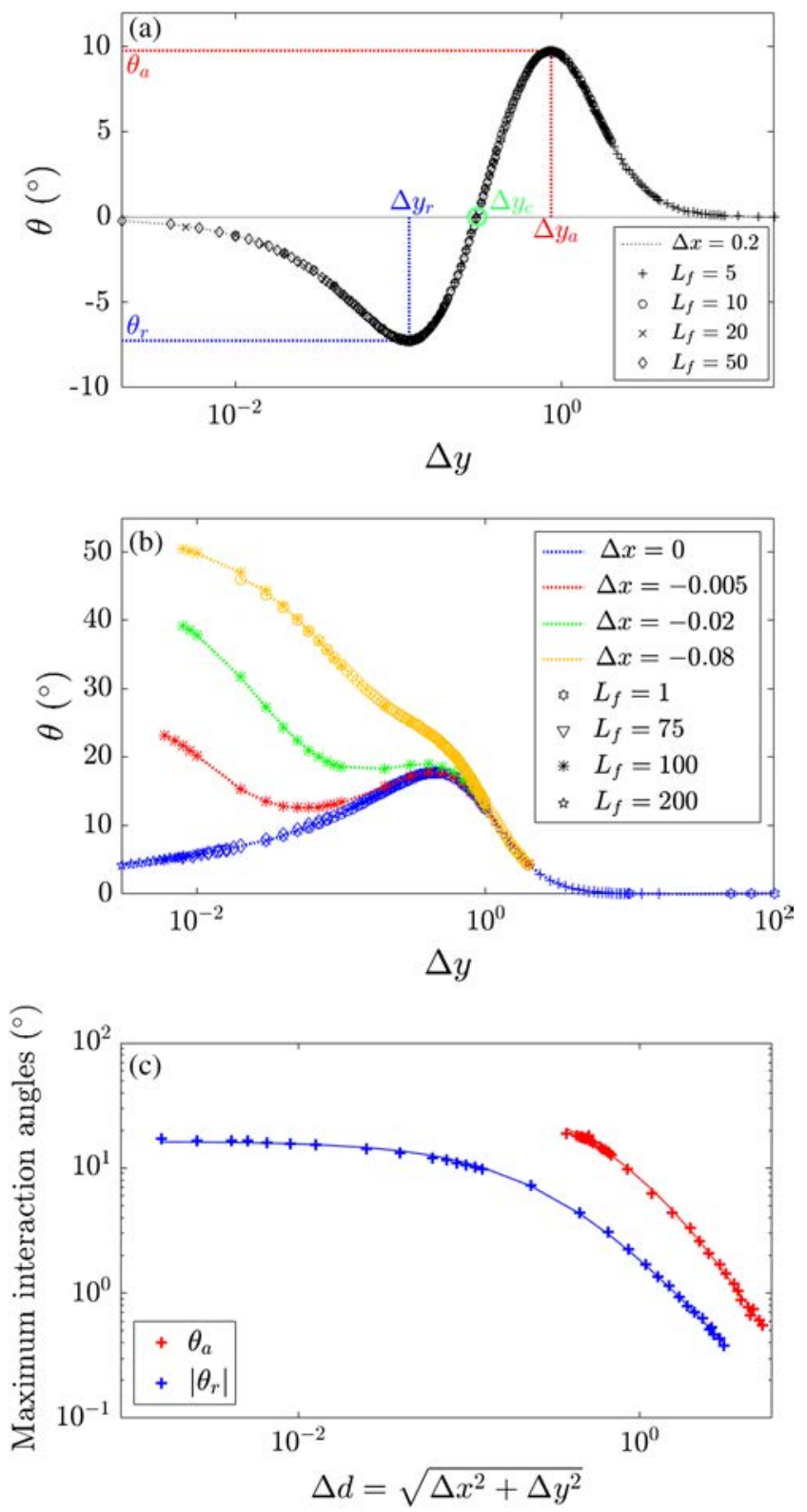

FIG. 2. (a) and (b) Typical $\theta(\Delta y)$ trend for approaching and superimposed cracks, respectively. Several crack lengths $L_{f}$ were used to reach a 5 orders of magnitude span in $\Delta y$. (c) Maximum interaction angles follow a shifted power law (continuous line) of the distance $\Delta d=\sqrt{\Delta x^{2}+\Delta y^{2}}$. Note that repulsion is recorded for much smaller tip to tip separation than attraction. 
$(\Delta x, \Delta y)$ points, therefore producing an accurate $\theta(\Delta x, \Delta y)$ map, the sign of $\theta$ indicating very clear repulsion and attraction zones.

An attractive to repulsive transition.-We find that the typical variation of $\theta$ with $\Delta y$ belongs to one of three kinds, depending on the fixed $\Delta x$ value; thus, we distinguish between approaching cracks $(\Delta x>0)$, coincident cracks $(\Delta x=0)$, and partially superimposed cracks $(-1<\Delta x<0)$.

In the case of approaching cracks, we retrieve the nonmonotonic behavior shown in Fig. 2(a). As expected, large lateral separations $\Delta y$ result in weak interaction; $\theta$ starts small and increases to convey increasing attraction as $\Delta y$ diminishes. Surprisingly, interaction does not intensify indefinitely as the cracks get closer: $\theta$ reaches its maximum value $\theta_{a}(\Delta x)$ at $\Delta y_{a}(\Delta x)$. After this point, $\theta$ decreases acutely to the extent of crossing the abscissa axis at $\Delta y_{c}(\Delta x)$ : the behavior becomes then exclusively repulsive. The existence of an optimum of repulsion $-\theta_{r}$ realized at $\Delta y_{r}$ comes off as a second surprise: amazingly, the magnitude of the interaction only decreases after this point until it reaches zero for perfectly aligned cracks. In the case of coincident cracks $(\Delta x=0)$ and partially superimposed cracks $(-1<\Delta x<0)$, the inner kink angle is positive for all values of $\Delta y$ : the cracks always exhibit an attractive behavior. In Fig. 2(b), we plotted the evolution of the initial kink angle when $\Delta y$ tends toward zero or, alternatively, when $L_{f}$ approaches infinity. While the repulsive zone disappears abruptly for $\Delta x \leq 0$, the optimum of attraction evolves continuously across $\Delta x=0$ : $\theta_{a}$ continues to exist for superimposed cracks not as a global maximum of attraction but as a local one. As the level of superimposition increases, $\theta_{a}$ progressively vanish until $\theta(\Delta y)$ becomes a purely decreasing function. It is possible to reach much larger kink angles in this situation: we recorded $\theta$ values up to about $55^{\circ}$ for largely superimposed cracks.

Phase diagram of crack interaction.-The various configurations are summarized in Fig. 3 showing the value of $\theta$ in the $(\Delta x, \Delta y)$ space. The landscape formed by the $\theta$ values presents multiscale characteristics. First, we find that the positions of the local extrema of attraction and repulsion, as well as the neutral line $\theta=0$, are reasonably fitted as power laws of $\Delta x$ (see also Table I):

$$
\Delta y_{i}=\Delta y_{i}^{0}+A_{i}|\Delta x|^{\alpha_{i}}
$$

The local optimum angles of interaction $\theta_{a}$ and $\theta_{r}$ form the crests and valleys of the landscape in Fig. 3. Their values can be fitted as shifted power laws [29] of the tip to tip distance $\Delta d=\sqrt{\left.\Delta x^{2}+\Delta y^{2}\right)}$ [Fig. 2(c)]:

$$
\theta_{a / r}=\theta_{0 a / r}\left(1+\frac{\Delta d}{\lambda_{a / r}}\right)^{-\alpha_{a / r}}
$$

Equations (1) and (2) quantify how sensible interacting cracks are to the initial configuration and, in a larger sense, how difficult it is to determine the path of interacting
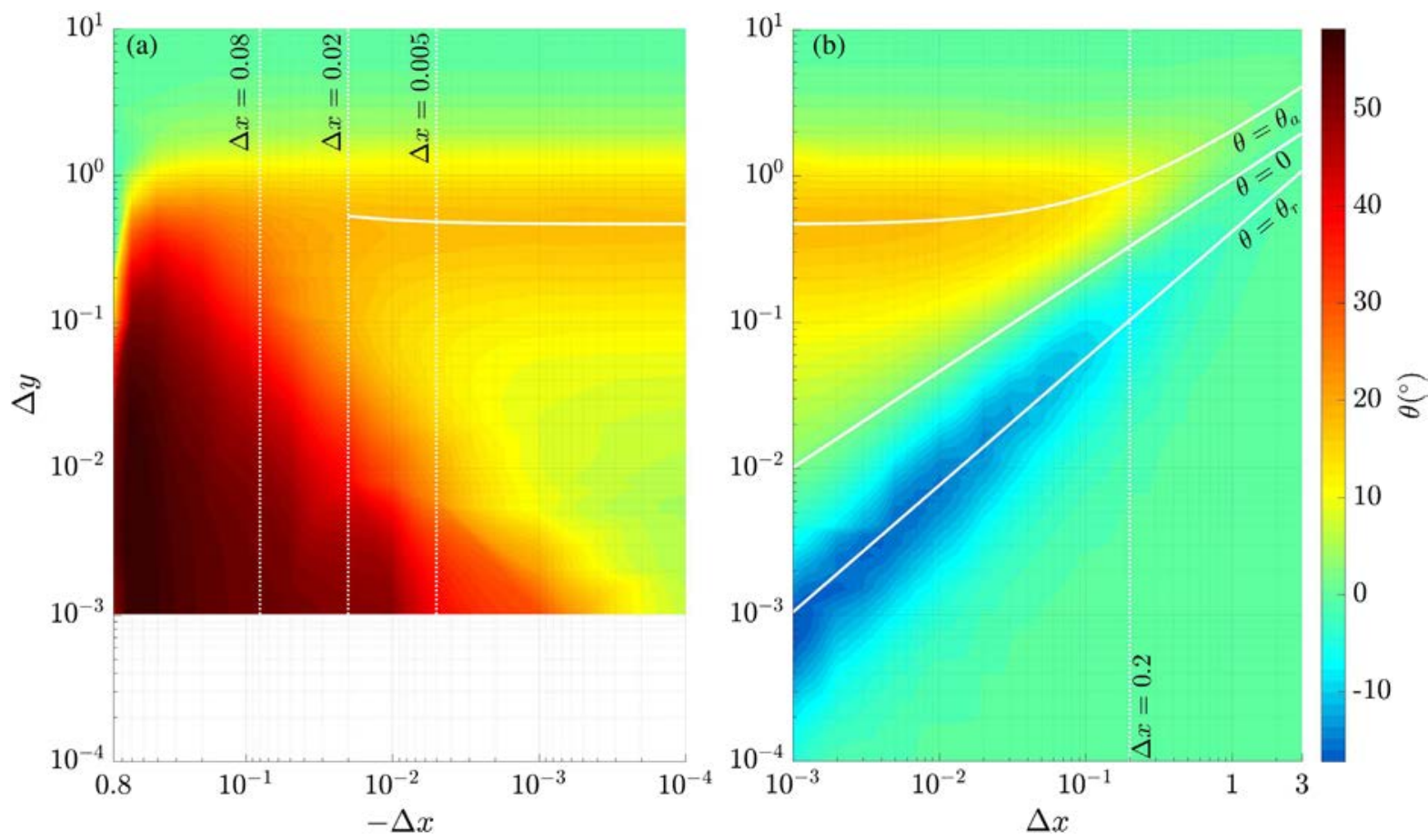

FIG. 3. Initial kink angle for overlapping cracks (a) and approaching cracks (b) in the ( $\Delta x, \Delta y)$ space. The superimposed white lines signal the local maxima of attraction and repulsion, as well as the transition from attractive to repulsive (defined by $\theta=0$ ). The dotted vertical lines show where the cuts for Figs. 2(a) and 2(b) were taken. 
TABLE I. Coefficients used in Eqs. (1) and (2) for the lines of maximum attraction or repulsion and the attraction-repulsion transition line. The nonzero $\Delta y_{a}$ indicates that an optimum of attractive behavior subsists well after superimposition of the inner tips.

\begin{tabular}{lllc}
\hline \hline & $\Delta y_{i}^{0}$ & $A_{i}$ & $\alpha_{i}$ \\
\hline$\Delta y_{a}$ & 0.45 & 1.5 & 0.77 \\
$\Delta y_{c}$ & 0 & 0.95 & 0.66 \\
$\Delta y_{r}$ & 0 & 0.41 & 0.86 \\
\hline \hline & $\theta_{0}$ & $\lambda$ & $\alpha$ \\
\hline$\theta_{a}$ & 44.8 & 0.96 & 2.35 \\
$\theta_{r}$ & -16.3 & 0.35 & 1.61 \\
\hline \hline
\end{tabular}

cracks. For $\Delta x>0$, both attraction and repulsion tend to become stronger when the crack tips are closer. The length scale $\lambda_{r}$ characterizing the increase in repulsion is, however, nearly 3 times smaller than the corresponding scale $\lambda_{a}$ for the attractive zone. Another remarkable scaling property is that attraction remains a dominant behavior when the vertical offset between the cracks $\Delta y$ is of the order of the crack length (as shown by $\Delta y_{a}^{0} \neq 0$ ), while repulsion becomes prevailing for crack tip distances corresponding to very small fractions of the crack length, typically of the order of $L_{f} / 100$ [Fig. 2(c)].

Concluding remarks.-In agreement with the computation reported by Dalbe et al. [17], we find that perfectly aligned cracks $(\Delta y=0)$ do not interact at all and propagate straight ahead. This failure of the principle of the local symmetry criterion to predict strongly repulsive paths as observed in experiments turns out to be only apparent. Indeed, as shown in Fig. 2(a) the initial angle of deviation grows steeply with a slight increase in lateral spacing $\Delta y$ so that the smallest perturbation can lead to significant repulsion, favoring unstable crack paths. This is consistent with the theory proposed by Melin [11] where the author concluded that the smallest perturbation in collinear en passant cracks will force the cracks to deviate from their straight path.

Overall, our numerical study provides a deeper understanding of how tip to tip separation affects the initial kink angle. While previous theoretical models of en passant cracks either neglected the repulsive part of the paths [10] or conjectured that repulsion is a product of plasticity $[17,18]$, we find that the theoretical framework combining linear elastic fracture mechanics and the principle of local symmetry is sufficient to quantitatively predict significant repulsion, up to nearly $18^{\circ}$. This Letter is a first step to reconcile theory with observation: the difficulty of predicting crack repulsion in the context of linear elastic fracture mechanics does not come from a limitation of the model or of the bifurcation criterion, but from the very specific $(\Delta x, \Delta y)$ domain in which repulsion can initiate.
Our results highlight the need to take into proper consideration the length of the en passant cracks when studying them. As the repulsion behavior happens when the inner tips are separated by only a fraction of the crack length, it is more easily revealed in natural settings. For instance, our analysis could help in understanding why spreading centers observed in geological situations and involving hundreds of kilometers long ridges interacting on a scale of a few hundred meters, commonly exhibit a repulsive deviation of their trajectories before overlapping $[9,30,31]$. The ability to model at small scales the attraction-repulsion transition during propagation of en passant cracks is especially relevant for industrial applications that involve a control of cracking behavior such as in mechanical sensors [28] or stretchable electronics [27]. We demonstrated that any numerical model of en passant cracks must be able to account for two very different length scales: the cracks length and the scale at which crack repulsion occurs, typically more than 2 orders of magnitude smaller.

The robustness of our computation method allows by iteration to determine complete en passant crack trajectories [Fig. 1(c)]. Taking into account the entire history of the crack path will be part of future work in which we will analyze more physically realistic trajectories. Indeed, because they can interact and deflect one another from afar, the cracks growing towards each other and reaching coincident or partially superimposed configurations will never be rigorously straight. Nevertheless, simulations with straight cracks allowed us here to capture the main interaction mechanism that is determined by the relative positions of the closest cracks tips, without considering the influence of the full crack path history.

S. S. acknowledges support of the Russian Government with Grant No. 14.W03.31.0002. L. V. would like to thank Anne Tanguy and Stéphane Roux for fruitful discussions.

*loic.vanel@univ-lyon1.fr

[1] G. Irwin, J. Appl. Mech. 24, 361 (1957).

[2] R. L. Kranz, Int. J. Rock Mech. Min. Sci. Geomech. Abstr. 16, 37 (1979).

[3] P. Cortet, G. Huillard, L. Vanel, and S. Ciliberto, J. Stat. Mech. (2008) P10022.

[4] T. Tentler and V. Acocella, J. Geophys. Res. 115, B01401 (2010).

[5] J. Sempere and K. Macdonald, Tectonics 5, 151 (1986).

[6] A. Eremenko, S. Novikov, and A. Pogorelov, J. Appl. Mech. Tech. Phys. 20, 477 (1979).

[7] S. Bechtle, S. Habelitz, A. Klocke, T. Fett, and G. Schneider, Biomaterials 31, 375 (2010).

[8] G. W. Patterson and J. W. Head, Icarus 205, 528 (2010).

[9] V. Acocella, A. Gudmundsson, and R. Funiciello, J. Struct. Geol. 22, 1233 (2000).

[10] M. Fender, F. Lechenault, and K. Daniels, Phys. Rev. Lett. 105, 125505 (2010).

[11] S. Melin, Int. J. Fract. 23, 37 (1983). 
[12] G. Sih, Eng. Fract. Mech. 5, 365 (1973).

[13] E. Gdoutos, in Problems of Mixed Mode Crack Propagation, edited by G. Sih (Martinus Nijhoff Publishers, Leiden, 1984), Chap. 4, pp. 71-96.

[14] B. Cotterell and J. Rice, Int. J. Fract. 16, 155 (1980).

[15] R. V. Goldstein and R. L. Salganik, Int. J. Fract. 10, 507 (1974).

[16] R. Ghelichi and K. Kamrin, Soft Matter 11, 7995 (2015).

[17] M. J. Dalbe, J. Koivisto, L. Vanel, A. Miksic, O. Ramos, M. Alava, and S. Santucci, Phys. Rev. Lett. 114, 205501 (2015).

[18] J. Koivisto, M.-J. Dalbe, M. J. Alava, and S. Santucci, Sci. Rep. 6, 32278 (2016).

[19] F. Erdogan and G. C. Sih, J. Basic Eng. 85, 519 (1963).

[20] B. Cotterell, Int. J. Fract. Mech. 1, 96 (1965).

[21] M. Amestoy and J. Leblond, Int. J. Solids Struct. 29, 465 (1992).

[22] J. R. Rice, J. Appl. Mech. 35, 379 (1968).

[23] M. Gosz and B. Moran, Eng. Fract. Mech. 69, 299 (2002).
[24] X. Suo and A. Combescure, Int. J. Fract. 57, 127 (1992).

[25] See Supplemental Material at http://link.aps.org/ supplemental/10.1103/PhysRevLett.120.255501, the complete computation scheme is presented along the equations necessary to compute $K_{I I}^{*}(\theta)$. Comparaison of our results with previous papers serves as validation of our method.

[26] K. J. Koester, J. W. Ager, and R. O. Ritchie, Nat. Mater. 7, 672 (2008).

[27] S. Lacour, J. Jones, S. Wagner, T. Li, and Z. Suo, Proc. IEEE 93, 1459 (2005).

[28] D. Kang, P. V. P., Y. Choi, C. Lee, S. Shin, L. Piao, B. Park, K. Suh, T. Kim, and M. Choi, Nature (London) 516, 222 (2014).

[29] J. L. Liu, J. Wang, Z. G. Yu, and X. H. Xie, Sci. Rep. 7, 1 (2017).

[30] K. MacDonald and P. Fox, Nature (London) 302, 55 (1983).

[31] D. D. Pollard and A. Aydin, J. Geophys. Res. 89, 10017 (1984). 